\title{
Electro-optical Detection of Charged Particle Beams*
}

\author{
Y.K. Semertzidis ${ }^{\dagger}$, V. Castillo, R.C. Larsen, D.M. Lazarus, B. Magurno ${ }^{\ddagger}$, T. Srinivasan-Rao, \\ T. Tsang, V. Usack, Brookhaven National Lab. \\ L. Kowalski, Montclair State Univ. \\ D.E. Kraus, Univ. of Pittsburgh
}

\section{Abstract}

We have made the first observation of a charged particle beam by means of its electro-optical effect on the propagation of laser light in a birefringent crystal at the Brookhaven National Laboratory Accelerator Test Facility. Polarized infrared light was coupled to a $\mathrm{LiNbO}_{3}$ crystal through a polarization maintaining fiber of 4 micron diameter.

An electron beam in $10 \mathrm{ps}$ bunches of $1 \mathrm{~mm}$ diameter was scanned across the crystal. The modulation of the laser light during passage of the electron beam was observed using a photodiode with $45 \mathrm{GHz}$ bandwidth. The fastest rise time measured, $120 \mathrm{ps}$, was made in the single shot mode and was limited by the bandwidth of the oscilloscope and the associated electronics. Both polarization dependent and polarization independent effects were observed. This technology holds promise of greatly improved spatial and temporal resolution of charged particle beams.

\section{INTRODUCTION}

A collaborative effort has been initiated to develop an ultrafast charged particle detector based on the birefringence induced in an optical fiber carrying polarized light due to the electric field of a relativistic charged particle. An analysis of such a detector is described in [1]. The electrooptical effect in amorphous optical media is known as the Kerr effect [2] and is quadratic in the electric field $E$; $\phi=2 \pi K E^{2} d$, where $\phi$ is the ellipticity induced in the polarized light, $K$ is the Kerr coefficient and $d$ is the length of the electric field region experienced by the material. In uniaxial crystals the induced ellipticity is linear in the externally applied E-field and the effect is called Pockels effect [3]. The induced phase delay is then given by $\phi=\pi\left(V / V_{\pi}\right)$ with $V$ the applied voltage and $V_{\pi}$ the voltage required for producing a phase shift equal to $\pi \mathrm{rad}$. As a first step towards realizing the single particle detector we used an intense, short length, electron beam from the Accelerator Test Facility (ATF) of Brookhaven National Lab (BNL) and the Pockels effect to detect it by optical means.

\section{SETUP AND SENSITIVITY}

The experimental setup in Fig. 1 shows the laser (CW, from Amoco Laser Company) with $20 \mathrm{~mW}$ of optical power in the infra-red $(\lambda=1.32 \mu \mathrm{m})$, polarized by the polarizer $(\mathrm{P})$

\footnotetext{
* Work supported in part by the U.S. Department of Energy under Contract N0. DE-AC02-98CH10886.

† Email: semertzidis@bnl.gov

$\ddagger$ Deceased
}

and coupled to the fiber $(F)$ with the microscope objective (L). The fiber is polarization maintaining with a core of $4 \mu \mathrm{m}$ in diameter.

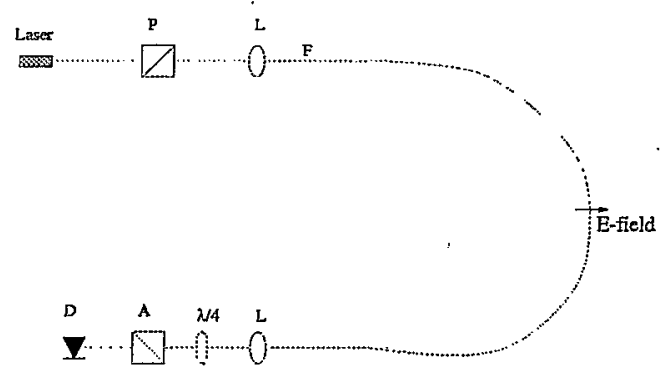

Figure 1: The experimental setup for detecting charged particle beams via optical means. The $\mathrm{LiNbO}_{3}$ crystal was located at the beam position indicated by E-field. The positions of the polarizer $(\mathrm{P})$, lenses $(\mathrm{L})$, analyzer $(\mathrm{A})$ and photodiode detector(D) are schematically indicated.

The fiber was coupled to a commercially available $\mathrm{LiNbO}_{3}$ crystal [4] as indicated in Fig. 1. The crystal package was modified to allow for the passage of a charged beam without hitting the housing. The laser light was extracted from the fiber and its polarization state analyzed with by means of a $\lambda / 4$ plate and the analyzer (A). It was then detected by the photodiode and pre-amplifier [5] the output of which goes to a fast transient digitizer [6].

The transmitted light from the analyzer is equal to

$$
I=I_{0}\left[\sigma^{2}+(\alpha+\phi(t))^{2}\right] \approx I_{0}\left[\sigma^{2}+\alpha^{2}+2 \alpha \phi(t)\right],
$$

with $I_{0}$ the light intensity before the analyzer, $\sigma^{2}$ the minimum possible ratio of $\left(I / I_{0}\right)$ when $\alpha$ and $\phi$ are equal to zero, $\phi$ the induced ellipticity, and $\alpha$ is an intentional misalignment angle introduced to linearize and amplify the effect. As is apparent from Eq. 1, the time dependent part of the light signal can be made positive or negative depending on the sign of $\alpha$ relative to $\phi$.

The ATF produced an electron beam of $45 \mathrm{MeV}$ kinetic energy, containing up to $1 \mathrm{nC}$ in $10 \mathrm{ps}$ bunches of $1 \mathrm{~mm}$ in diameter and a repetition rate of $1.5 \mathrm{~Hz}$. This charged particle beam creates an electric (E) field at a distance $r$ if $r \gg$ than the dimensions of the beam bunch:

$$
E=\gamma N_{e} \frac{q}{4 \pi \epsilon_{0} r^{2}}=\gamma N_{e} \times 5.8 \times 10^{-5} \mathrm{~V} / \mathrm{m},
$$

with $\gamma$ the relativistic Lorentz factor, $N_{e}$ the number of electrons in the beam, $q=1.6 \times 10^{-19} \mathrm{C}$ the electron 
charge, $\epsilon_{0}=8.85 \times 10^{-12} \mathrm{~F} / \mathrm{m}$ the permittivity of free space, and $r$ the distance from the material (in the example we used $r=0.5 \mathrm{~cm}$ ). This electric field is present for

$$
\Delta t=\frac{r}{\gamma u}=\frac{17}{\gamma} \mathrm{ps},
$$

with $u$ the beam velocity.

The $\mathrm{LiNbO}_{3}$ crystal used has $V_{\pi}=5 \mathrm{~V}$ with an electrode separation of $15 \mu \mathrm{m}$ and a length of $l=1.5 \mathrm{~cm}$. The integral then of $\int E d l=\int \frac{5 \mathrm{~V}}{15 \times 10^{-5} \mathrm{~m}} d l=5000 \mathrm{~V}$ is capable of producing $\pi \mathrm{rad}$ of phase shift or $\pi / \sqrt{2} \mathrm{rad}$ maximum of ellipticity (the maximum ellipticity is induced when the laser polarization is at $45^{\circ}$ with respect to the applied electric field direction). The same integral estimated for a particle beam located at the mid-plane orthogonal to the crystal at a distance $r=0.5 \mathrm{~cm}$ is

$$
\int E d l=\frac{\gamma N_{e} q}{4 \pi \epsilon_{0} r} 2\left(1-\sin \theta_{1}\right)=\gamma N_{e} 2.6 \times 10^{-7} \mathrm{~V}
$$

with $\theta_{i}$ the angle $A \hat{B C}$ where $A$ is the location of the beam, $B$ one end of the crystal in the long direction and $C$ the center of the crystal. This produces an ellipticity of

$$
\phi=\gamma N_{e} \times 0.1 \mathrm{nrad},
$$

for $17 / \gamma \mathrm{ps}$.

The signal to noise ratio $(S N R)$ for a detection system which is photon statistics limited is given by

$$
S N R=\phi \sqrt{\frac{P T q_{p}}{2 \hbar \omega}},
$$

with $P$ the laser power, $T$ the inverse of the detection system bandwidth, $q_{p}$ the quantum efficiency of the photodiode, and $\hbar \omega$ the energy of the laser photon. As an example we will take an electron particle beam with $\gamma=1700$, $T=10 \mathrm{fs}, q_{p}=0.8, P=10^{8} \mathrm{~W}$ (e.g. $1 \mathrm{~mJ}$ pulsed laser light for $10 \mathrm{ps}$ ) and $\hbar \omega=0.9 \mathrm{eV}$, then the required number of electrons in the beam for $S N R=1$ is $N_{e} \sim 2$.

\section{EXPERIMENTAL RESULTS}

At the experimental setup we used a CW laser of $10 \mathrm{~mW}$, and a detector with $100 \mathrm{ps}$ time resolution. Then using the above Eqs. 3, 6 we estimated the number of particles needed in the electron beam to be $N_{e}=10^{8}$ for $S N R=1$. Our beam of $1 \mathrm{nC}$ corresponds to $N_{e}=9 \times 10^{9}$ which ensured its detection. The induced ellipticity (from Eq. 5) is $\phi=1.0 \gamma \mathrm{rad}$ equivalent to $\phi=0.2 \mathrm{rad}$ when the signal attenuation due to limited detector bandwidth is taken into account.

In Fig. 2 we show the polarization dependent signal (solid line) as observed with a single shot of the electron beam. Changing the sign of $\alpha$ (see Eq. 1) the signal also flips sign while retaining the same amplitude. The maximum modulation of the light intensity was about $9 \%$ of its DC level.

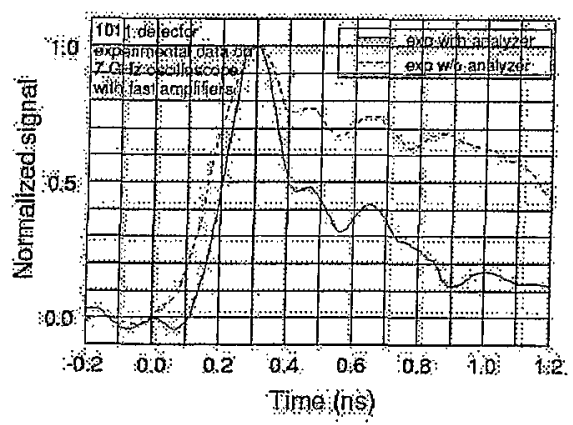

Figure 2: The polarization dependent signal (solid line). The electron beam was about $r=0.5 \mathrm{~cm}$ from the crystal. The polarization independent signal is also shown (dash line).

We have also observed a signal when the crystal intercepted the beam which is shown in Fig. 2 again in the single shot mode (dash line). We repeated this without the analyzer present and found it was independent of it. The difference from the polarization dependent signal is two-fold. First it does not flip sign under any polarization orientation, and second it has a much longer time decay constant.

We intend to improve the time resolution of the signal by increasing the laser light intensity and the oscilloscope bandwidth. Long term plans include the implementation of this sensitive method as a readout to a high rate single particle detector.

If $r$ is reduced to $100 \mu \mathrm{m}$, the required $N_{e}$ is reduced by a factor of 2 , making possible ultra-fast single particle detection by optical means. The gain factor is only 2 because of the limited bandwidth of the assumed detector which attenuates the signal by the ratio of $\Delta t / T$.

We will also look into a new type of fiber which exhibits high polarizability [7] thus reducing the cost of the detector considerably.

\section{REFERENCES}

[1] Y.K. Semertzidis, XXVII Intemational Conference on High Energy Physics gls0918 (1994).

[2] J. Kerr, Phi. Mag. 50, 337, 446 (1875).

[3] A. Yariv, Quantum Electronics, Wiley, New York, 1967, 3rd ed. 1989.

[4] Uniphase Telecommunications Products, 1289 Blue Hills Ave., Bloomfield, CT 06002.

[5] Type 1011 pre-amplifier from New Focus, Inc., 2630 Walsh Ave., Santa Clara, CA 95051. The conversion gain of the detector is $10 \mathrm{~V} / \mathrm{W}$, with a rise time of $9 \mathrm{ps}$.

[6] The oscilloscope used was from Hewlett Packard (HP SDC5000), of $7 \mathrm{GHz}$ bandwidth.

[7] X.C. Long and S.R.J. Brueck, IEEE Photonic Technology Letters, vol 9, p.767, 1997. 